\title{
DIGITAL DESIGN AND SIMULATION OF CVSD CODEC ALGORITHM USING VHDL
}

\author{
Amrita Soni ${ }^{1}$, Piyush Gupta ${ }^{2}$ \\ ${ }^{1}$ Asst. Professor, Department of Electronics and Communication Engineering, UEC, Ujjain, (M.P.), India \\ ${ }^{2}$ M.E. Research Scholar Department of Electronics and Communication Engineering UEC, Ujjain, (M.P.), India
}

\begin{abstract}
Generally, digital signal transmission systems have important advantages over analog counterparts because noise dose not built up in cascading of repeaters. There is various digital transmission techniques like PCM, DM, DPCM, ADM etc. In this DM and ADM provides minimum transmission Bandwidth. DM provides slope overload error and granular noise in transmission so variation of step size is needed, which is provided by ADM. There is various algorithms in ADM technique like SONG algorithm, ABATE algorithm etc. one of the algorithm of ADM is CVSD algorithm. CVSD (continuous variable slope delta) modulation is an effective scheme for audio signal. CVSD is best coding technique for improving receiver sensitivity with low transmission rate as compare to PCM. Here in this paper, we discuss digital design of CVSD codec system using VHDL and simulation result is obtained at various data rates.
\end{abstract}

Keywords: CVSD, DM, PCM, FPGA, ASIC, VHDL, BW, SIPO, PIPO, Verilog, RTL.

\section{INTRODUCTION}

Continuously variable slope delta modulation (CVSD or CVSDM) is an audio encoding method. It is a delta modulation with variable step size [4]. Due to variation in step size slope overload error is decreases as compare to delta modulation. The CVSD modulation is a technique of digitizing a band-limited audio signal. The CVSD modulator is many to one bit compression technique, which compresses the 8 bit into 1 bit or 16 bit into 1 bit or 12 bit data into one single bit. It is an lossy compression technique. The output of this 1-bit encoder is a serial bit stream, where each bit represents an incremental increase or decrease in signal steps amplitude. The continuously variable slope delta (CVSD) modulation is a nonlinear [1], sampled data, closed loop system which accepts a band-limited analog signal and encodes it into binary form for transmission in a digital channel. At the receiver, the binary signal is decoded into a close approximation of the original analog signal. Continuously Variable slope Delta Modulation is a simple s step adaptive DM method offering low hardware complexity, improved noise performance and minimum transmission Bandwidth [3]. It is an attractive alternative to more complex conventional analog to digital signal conversion techniques used in telecommunication and signal processing. To implement CVSD, There are few CVSD chip sets such as MC34115 from Motorola [2] but it works only at $16 \mathrm{kbps}$ and it does not allow to add encryption to the algorithm, which is very important in defense systems for security purposes. Furthermore, to implement such algorithms with encryption possible, software (i.e. programming in DSP/GPP) and hardware (i.e. programming in FPGA or ASIC i.e. programming in VHDL or Verilog) approaches are available.

\section{RTL SCHEMATIC OF CVSD CODEC IN} \section{QURTUS-II}

The CVSD modulator consist an 8 bit comparator for comparing $\mathrm{i} / \mathrm{p}$ signal with reference signal, 3 bit sipo(serial in parallel out) for detecting slope overload, 8 bit pipo(parallel in parallel out) for providing 1 clock cycle delay, overload detect and level select algorithm is used for selecting the variable step size. The present input compared with previous level selected output in 8 bit comparator. The comparator output is a digital encoded output which is 1 bit compressed output of 8 bit A to D input. Here shows the RTL view of CVSD TOP Module designed in altera quartus II.

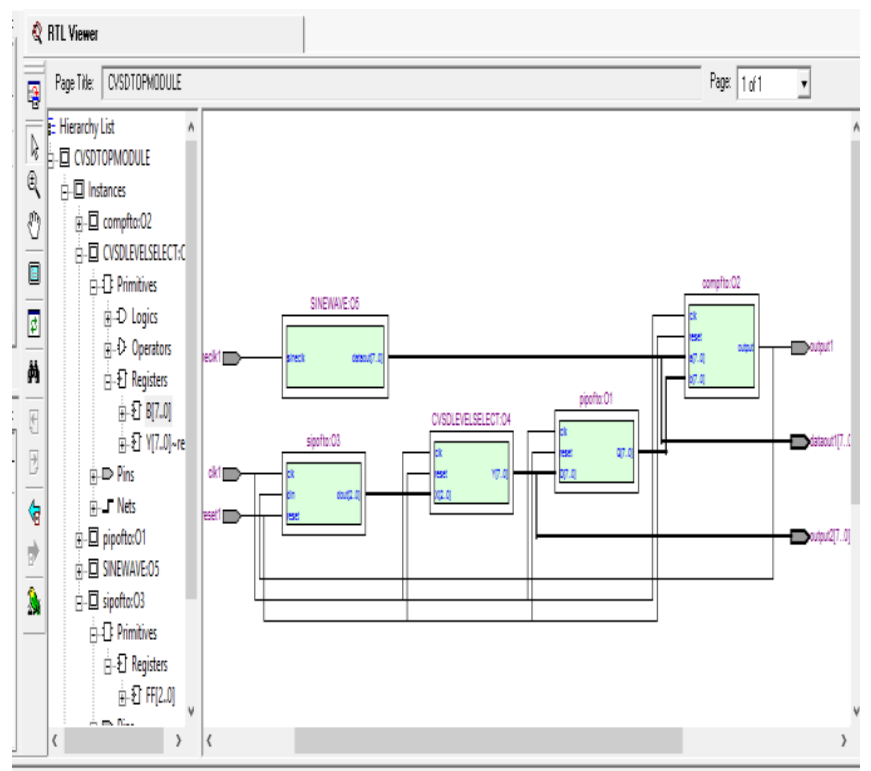

Fig-1.RTL view of CVSD top module 


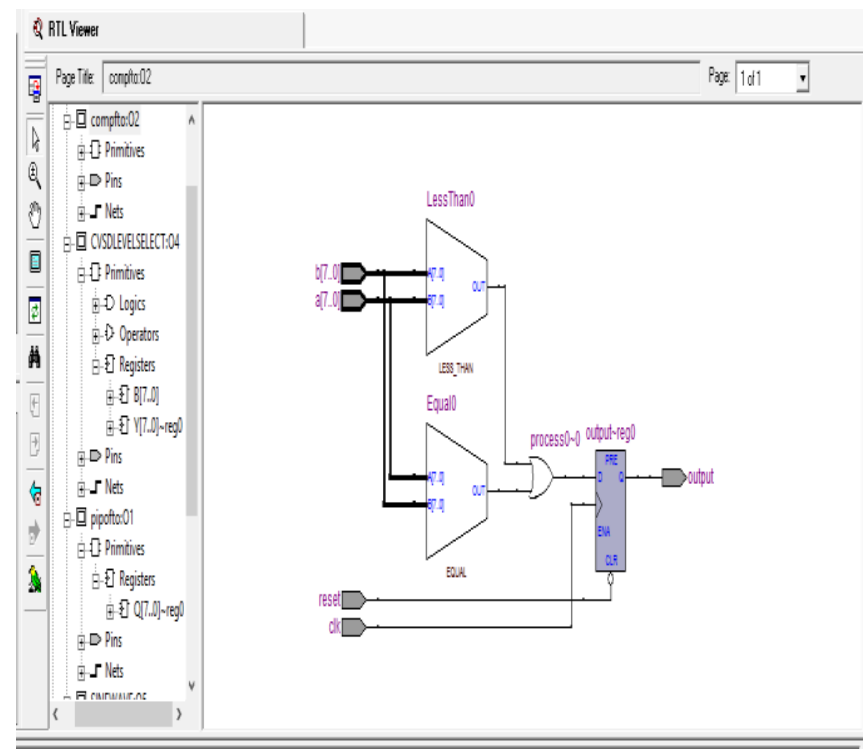

Fig-2 RTL view of 8 bit comparator

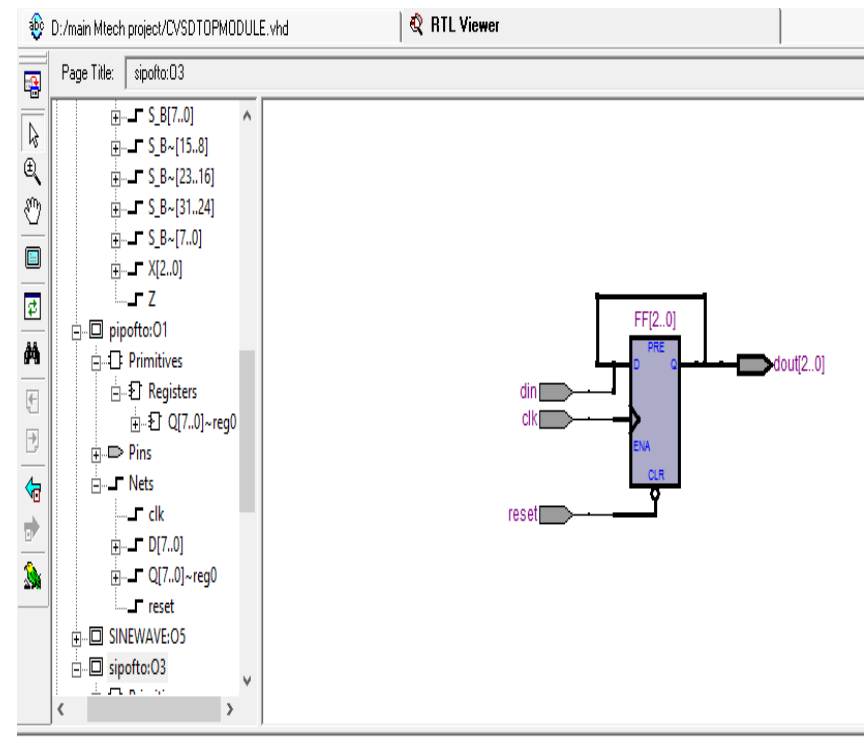

Fig-3 RTL view of 3 bit sipo register

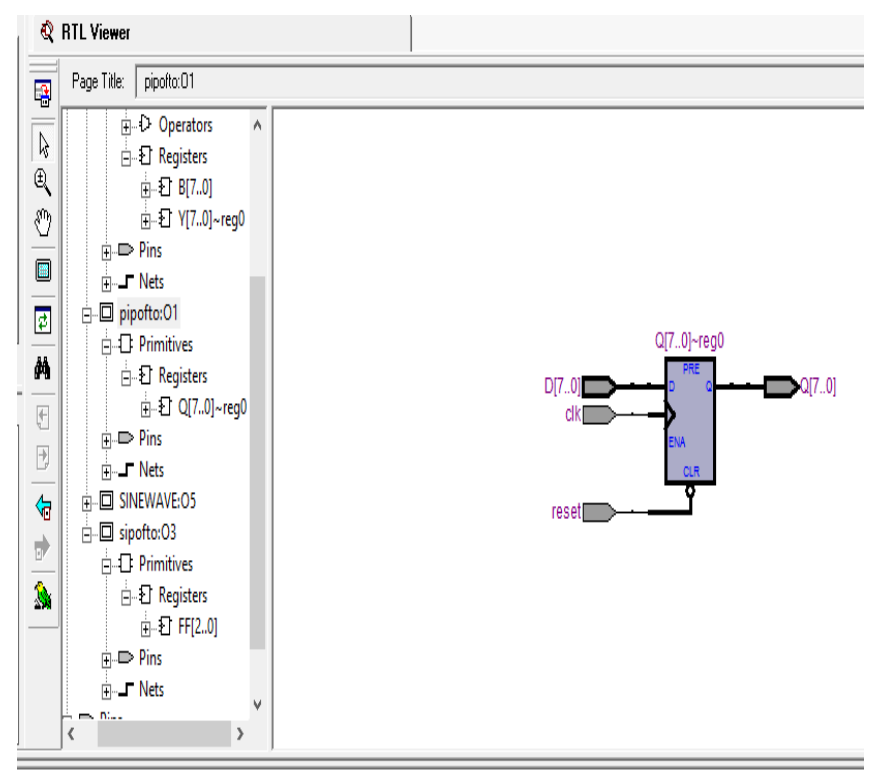

Fig-4 RTL view of 8 bit pipo register

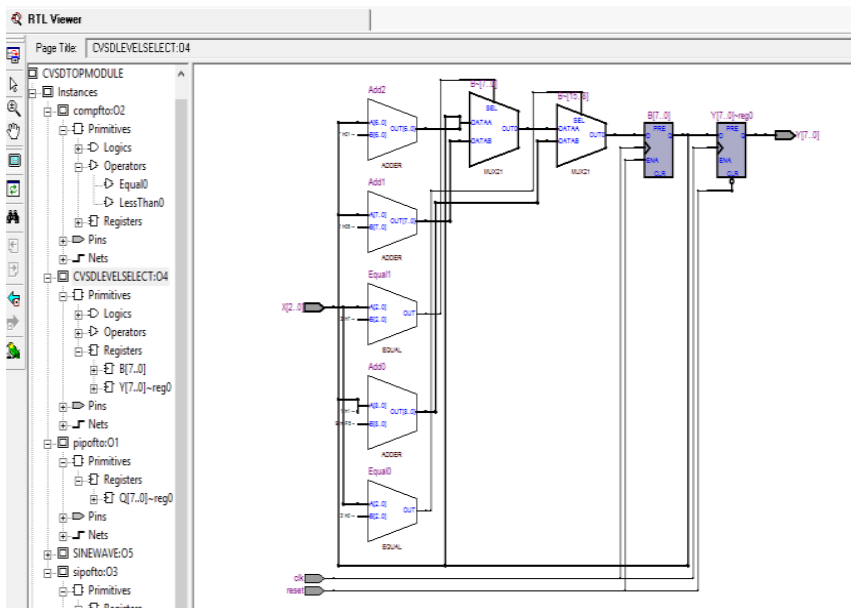

Fig-5 RTL view of CVSD level select module

The CVSD demodulator consist an 3 bit sipo(serial in parallel out) register, overload detect and CVSD level select as shown in figure 2 . The 3 bit sipo take 3 bit digital input and then this input is checked by overload detect block, according to the digital input the step size level is selected by level select algorithm. The level select output is the 8 bit Demodulated output which is decompressed 8 bit output. The algorithm used in both the modulating side and demodulating side are same. Here above figure shows the RTL schematic of various modules created in quartus-II in VHDL.

\section{CVSD ENCODER AND DECODER}

\section{ALGORITHM}

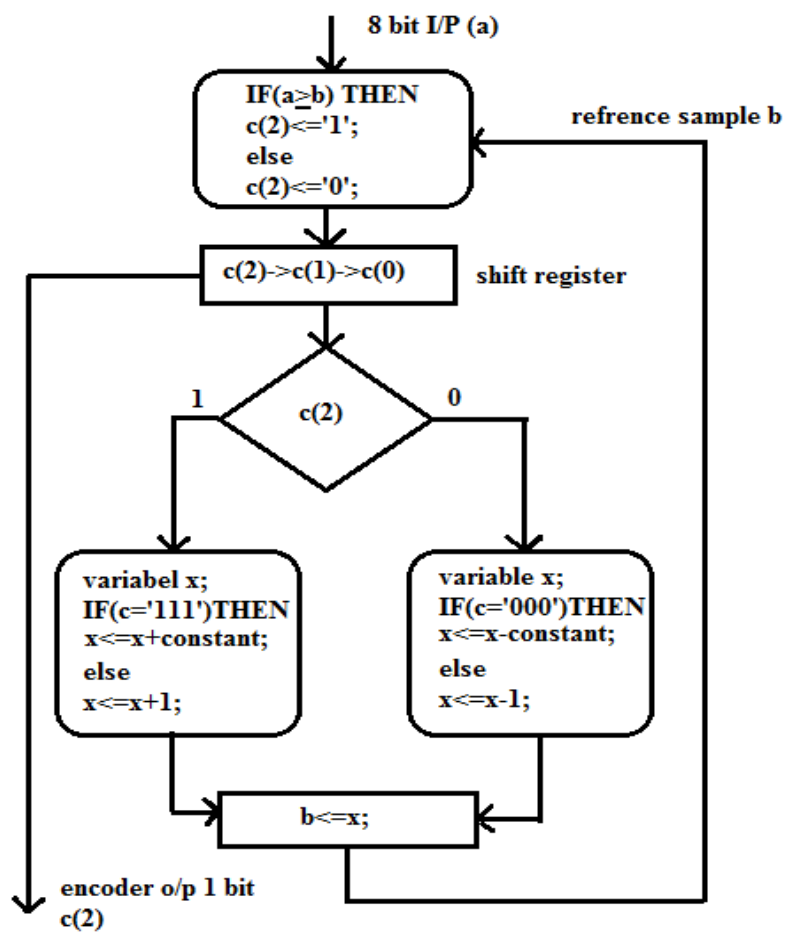

Fig-6 CVSD Encoder algorithm 
Here Figure 6 shows the CVSD encoder algorithm. Here in this process in first step, the I/O 8 bit data is compare with the reference sample 8 bit magnitude. Then if the reference sample is less then $\mathrm{I} / \mathrm{P}$ then output is ' 0 ' otherwise $\mathrm{O} / \mathrm{P}$ of the comparator is ' 1 '. In second step the output of comparator is input of 3 bit shift register. Here the MSB bit output of shift register is encoded 1 bit output, which is 8 to 1 bit compressed output. In the step 4 , the reference sample ' $b$ ' is taken from CVSD level select algorithm. Here if shift register output is 111 or 000 then the slop of the input signal is continually increasing or continuously decreasing respectively, so reference output $b$ is increased or decreased by an arbitrary constant value (i.e. Increment of step size). Otherwise increment or decrement by one step.

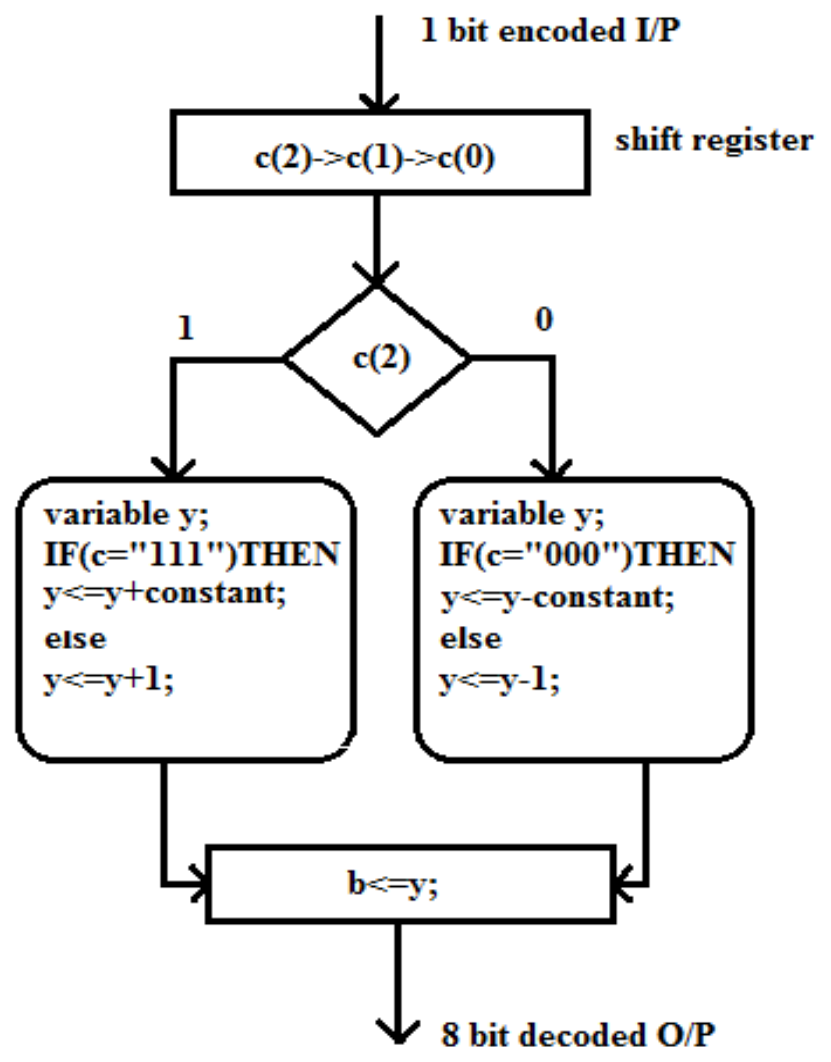

Fig-7 CVSD Decoder Algorithm

Here Figure 7 shows the CVSD decoder algorithm. In first step, encoder one bit output is input of 3 bit serial in parallel out shift register. If sipo register contain " 111 " or " 000 " then decoder analysis that there is continuously increment or decrement in slope of the input signal. So step size is increases or decreases by an arbitrary constant respectively. Otherwise increment or decrement by one step.

\section{SIMULATION}

Here in figure 8, 9 and 10 shows the waveform simulation at various data rates,

Sampling rate of input signal $=1 /(400 \times 30)=83.33 \mu \mathrm{s}$

$\mathrm{i} / \mathrm{p}$ signal frequency $=400 \mathrm{~Hz}$

grid size $=83.33 \mu \mathrm{s}$
Clock rate for $16 \mathrm{kbps}=1 /(1024 \times 16)=61.035 \mu \mathrm{s}$

Clock rate for $32 \mathrm{Kbps}=1 /(1024 \times 32)=30.51 \mu \mathrm{s}$

Clock rate for $32 \mathrm{Kbps}=1 /(1024 \times 64)=15.25 \mu \mathrm{s}$

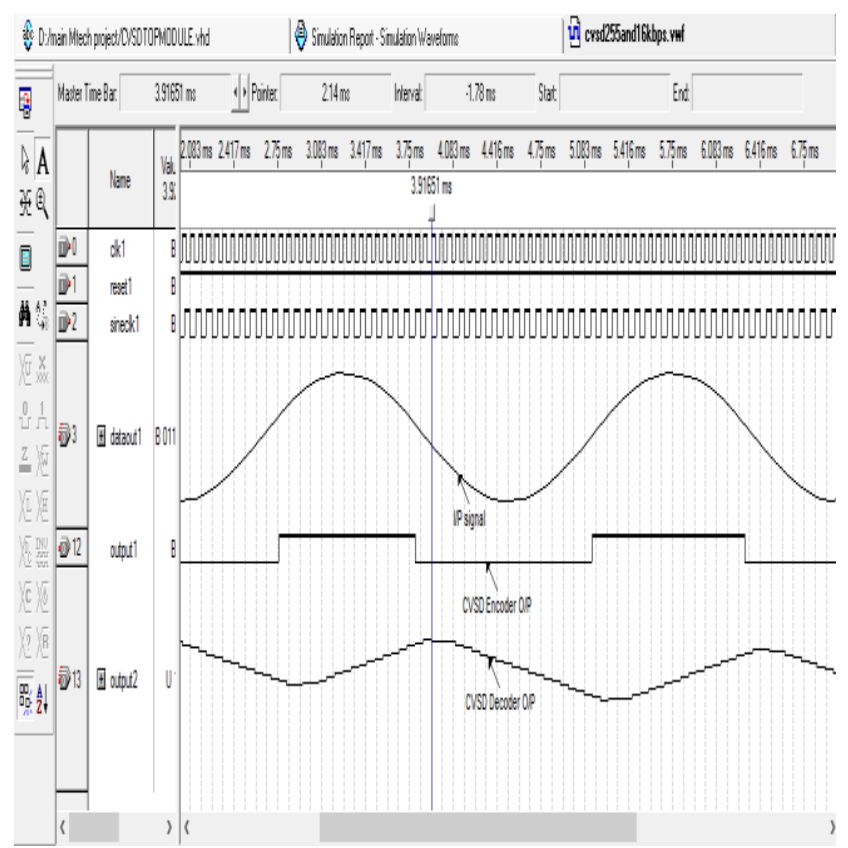

Fig-8 CVSD waveform simulations at $16 \mathrm{kbps}$ data rate

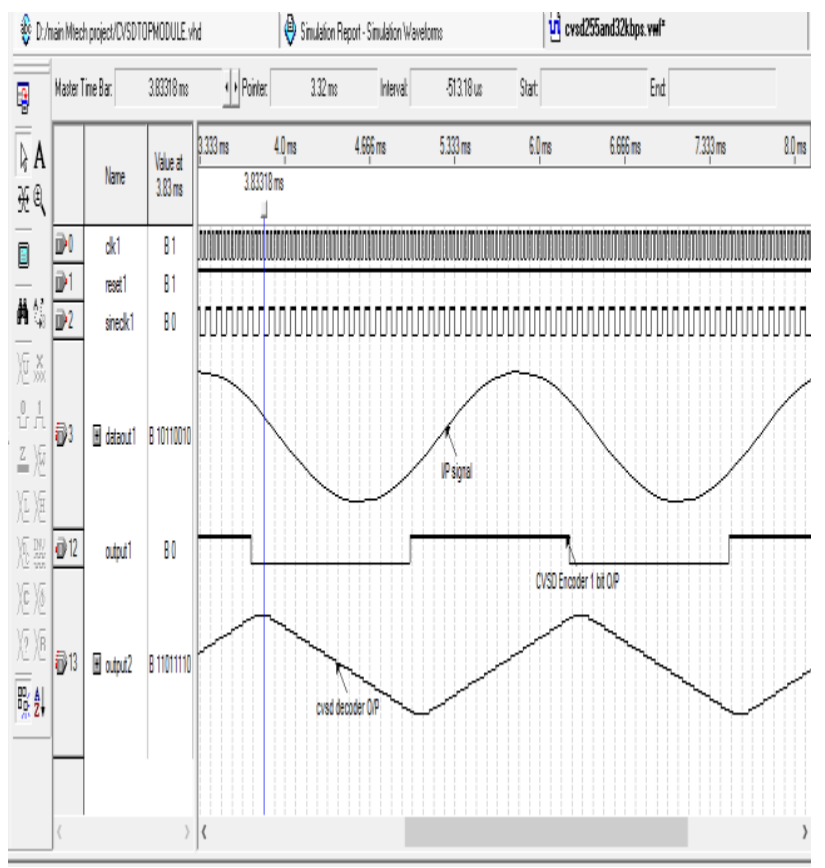

Fig-9 CVSD waveform simulations at $32 \mathrm{kbps}$ 


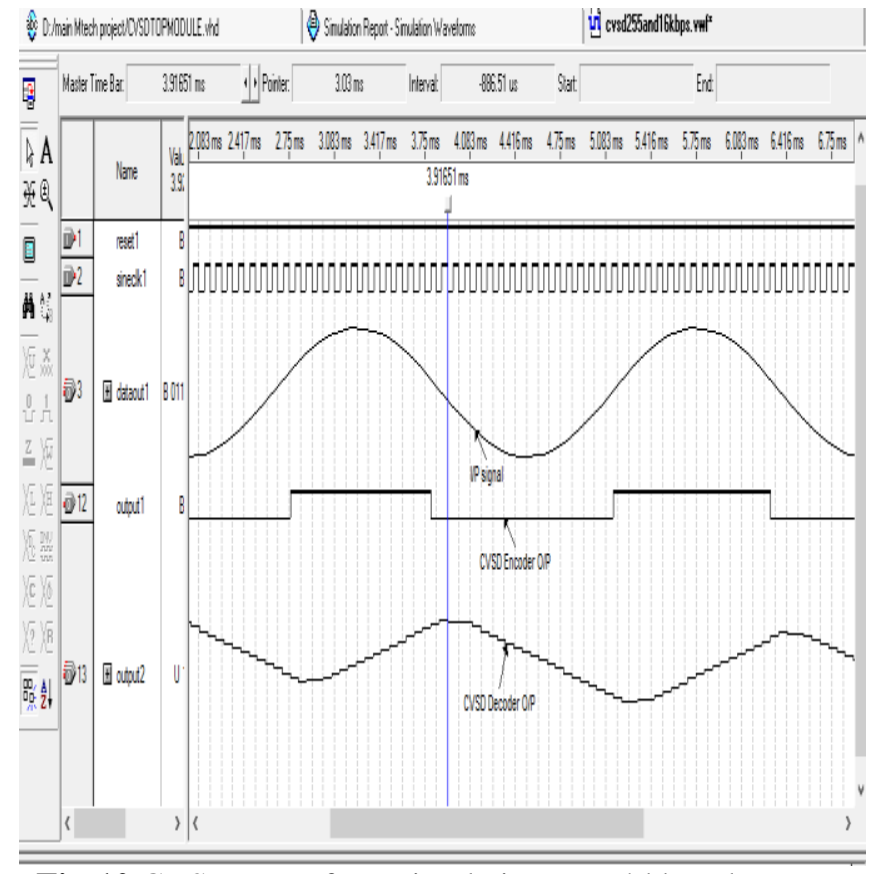

Fig-10 CVSD Waveform simulations at $64 \mathrm{kbps}$ data rate

\section{Graph between Data rate vs. SNR(DB)}

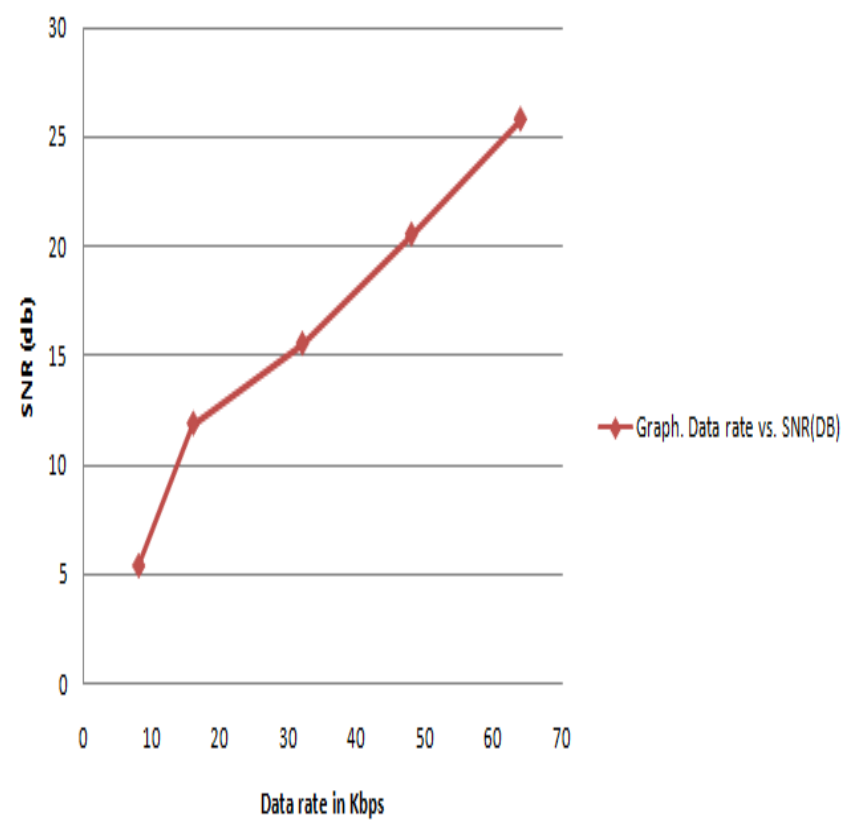

Fig-11 Graph between SNR vs Data Rate

Table-1 Data rate Vs SNR

\begin{tabular}{|l|l|}
\hline $\begin{array}{l}\text { Data Rate } \\
\text { (kbps) }\end{array}$ & SNR(db) \\
\hline 8 & 5.367 \\
\hline 16 & 11.8892 \\
\hline 32 & 15.5511 \\
\hline 48 & 20.5544 \\
\hline 64 & 25.824 \\
\hline
\end{tabular}

Here in above graph shows that by increasing the data rate SNR of cvsd is increases. The SNR calculation at different data rate is done by the following formula at sampling rate $83.33 \mu \mathrm{s}$.

$$
S N R=10 \log _{10} \frac{\text { variance }(\text { Input })}{\text { variance }(\text { Output }- \text { Input })}
$$

$$
\begin{aligned}
& \text { SNR }=10 \log _{10} \frac{\sum_{n=1}^{N}\left(x_{n}\right)^{2}}{\sum_{n=1}^{N}\left(x_{n}-\hat{x}_{\mathrm{n}}\right)^{2}} \quad \ldots . . .[7,8] \\
& \left\{x_{\mathrm{n}}\right\} \rightarrow \begin{array}{l}
\rightarrow \text { samples of original signal (speech } \\
\text { signal) }
\end{array} \\
& \left\{\hat{x}_{\mathrm{n}}\right\} \quad \rightarrow \text { samples of final reconstructed signal }
\end{aligned}
$$

\section{CONCLUSION}

Here, I described a brief introduction to continuous variable slope delta modulation and Algorithms of CVSD Encoder and Decoder. The main benefit of CVSD is that it reduces the slope overload error as compare to Delta Modulation because of step size is continuously changeable by CVSD algorithm. It is most efficient lossy compression technique for voice coding and decoding with less error. The CVSD codec eliminate the need of complex framing because of one bit encoding (i.e. compression from 8 bit to 1 bit and Decompression of 1 bit to 8 bit).

Here I also described the various SNR in DB at different data rates in kbps. As we increases the data rate of cvsd codec the SNR of the output is increased.

\section{REFERENCES}

[1]. B. Shiva Kumar Reddy, Lakshmi Boppana and Ashok Agarwal. "BER Analysis of CVSD Vocoder for WiMAX using GNU Radio". IEEE Region 10 Symposium, 2014 IEEE.

[2]. Hari Krishna Boyapati, Rajeev Kumar Elubudi, Manoj Jain. "Efficient Implementation of Voice Processing Module with CVSD Source Coding for Tactical wireless system".2015 IEEE International Conference on Research in Computational Intelligence and Communication Networks. [3]. G.S TOMBRAS and C. COURTIS. "Implementation and Performance of a Delayed Continously Variable Slope Delta Modulator". Electrotechnical Conference, 1991. Proceedings., 6th Mediterranean

[4]. Donald s. Taylor. "Design of continuously variable slope delta modulation". Motorola analog marketing.

[5]. B.P. Lathi \& Zing, Modern Digital \& Analog Communication System, Oxford University Press, International Fourth edition 2010. 
[6]. "Adaptive Delta Modulation Techniques" Niranjan U and M.N. Suma, International Confrence on Electronic Design and Signal Processing(ICEDSP) 2012.

[7]. "Study of a Novel ADM Algorithm with Pre-processing for Performance Improvement"B K Sujatha1, Dr. P S Satyanarayana2, FIETE, Dr. K N Haribhat3 1M S Ramaiah Institute of Technology, Bangalore $2 \mathrm{~B}$ M S College of Engineering., Bangalore 3Nagarjuna College of Engineering and Technology, Bangalore

[8]. "implementation and performance of a delayed continuously variable slope delta modulator" 6.8.TOMBRAS and C.COURTI8 Inrtitutm of Informatics 8, Telecommunications. N.C.S.R. "Denokritos". GR-1S3 10 Athens. Grerce. 